\title{
In vivo three-dimensional analysis of distal radioulnar joint kinematics during forearm pronation-supination
}

\author{
Kiyoko KAZAMA*,**, Koichi KOBAYASHI** and Makoto SAKAMOTO** \\ * Department of Radiology, Niigata Hand Surgery Foundation Niigata Hand Care Center \\ 997 Suwayama, Seiro-machi, Kitakambara-gun, Niigata 957-0016, Japan \\ ${ }^{* *}$ Graduate School of Health Sciences, Niigata University \\ 2-746 Asahimachi-dori, Chuo-ku, Niigata 951-8518, Japan \\ E-mail: kobayasi@clg.niigata-u.ac.jp
}

Received 3 July 2015

\begin{abstract}
The action of the forearm is to transfer force and torque across the elbow and wrist, resulting in axial rotation at the distal and proximal radioulnar joints, called pronation and supination. Pronation and supination are important functions as they allow many kinds of activities of daily living. Three-dimensional (3D) motion analysis of pronation and supination is essential for a better understating of the biomechanics of the forearm to describe the physiological range of motion and optimize clinical options for disorders of the forearm. Although numerous in vitro studies have contributed to gain insights into the forearm pronation and supination, in vivo investigations are still limited. In this study, three-dimensional (3D) motion of the distal radioulnar joint (DRUJ) during forearm pronation and supination was analyzed in vivo using biplanar radiography and 3D models of the ulna and radius. Twelve healthy subjects ( 6 men and 6 women) were recruited for the study. Each subject's right forearm (dominant hand) underwent a computed tomography (CT) scan to create 3D surface models of the ulna and radius. Each subject was imaged using a calibrated biplanar radiographic system at $90^{\circ}$ pronation, $45^{\circ}$ pronation, neutral, $45^{\circ}$ supination, and $90^{\circ}$ supination while the elbow was in extension or flexion. The 3D positions of the radius and ulna were obtained using a 2D-to-3D image registration method. The relative translations of the radius in the radioulnar, dorsopalmar, and proximodistal directions and the relative rotations of the radius about the radioulnar, dorsopalmar, and proximodistal axes were evaluated with respect to the ulna during pronation and supination. In general, the radius translated towards the ulnar side throughout forearm rotation, and moved towards the palmar side as pronation increased and moved dorsally as supination increased, and was positioned proximally during pronation and distally during supination. The rotation of the radius was dominant about the proximodistal axis. These kinematic variables were affected by elbow position and sex of the subject. The current findings regarding the 3D kinematics of the DRUJ during forearm pronation and supination may contribute to further understanding of the physiological biomechanics of the upper limb.
\end{abstract}

Key words : Biomechanics, Forearm, Distal radioulnar joint, In vivo kinematics, Image matching

\section{Introduction}

The forearm consists of two parallel long bones, the radius and ulna, with multiple ligaments as stabilizers and two articulations, one at the proximal radioulnar joint (PRUJ) and the other at the distal radioulnar joint (DRUJ). The forearm transfers force and torque across the elbow and wrist resulting in axial rotation at the PRUJ and DRUJ, called pronation and supination. In addition to axial rotation, a translation or gliding movement can occur between the distal radial and ulnar articulating surfaces during supination and pronation as the curvature of the radial sigmoid notch is greater than that of the ulnar head (Kapandji, 2001). The range of motion (ROM) accomplished by pronation and supination exceeds $180^{\circ}$, which is thought to be higher in women than in men (Rickert, et al., 2008; Soucie, et al., 2011), and affected by the flexion of the elbow joint (Kapandji, 2001). This large ROM facilitates many kinds of 
activities of daily living, leisure, and sports, such as lifting weights, turning doorknobs, manipulating tools, swinging tennis rackets, and so forth. Disorders of the DRUJ with reduced ROM can thus considerably impede these activities.

Instability is one of the common causes of dysfunction at the DRUJ. The distal radioulnar joint instability may result from a distal radial fracture, a traumatic load exerted after a fall on an outstretched hand, or degenerative change at the articulating surface. In addition, a morphological and biomechanical study has reported that individual anatomical differences such as the ulnar variance and the ulnar foveal shape have an impact on the kinematics of the DRUJ (Kataoka, et al., 2012). Therefore, investigating the normal and pathological motions of the DRUJ is crucial to understanding the biomechanics of the forearm. Numerous studies on forearm kinematics have been carried out both in vitro and in vivo (Stuart et al., 2000; Shaaban et al., 2004; Ray, et al., 1951). Recent progress in imaging techniques, in particular, allows three-dimensional (3D) analysis in vivo. Utilizing computed tomography (CT), the axis of forearm rotation (Tay, et al., 2007; 2010, Kataoka, et al., 2012) and the gliding and contact characteristics at the articulating surfaces (Tang and Chen, 2012; Chen and Tang 2013) have been evaluated. Nakamura et al. (1999) pioneered research in magnetic resonance imaging (MRI) of forearm rotation. Also, a two-dimensional (2D)-to-3D image-matching approach, which registers the 3D positions of bone models by matching their silhouettes with radiographic images, has been used to capture the in vivo 3D kinematics of several kinds of joints. Using this methodology, the location of the forearm rotation axis as well as translation of the ulnar head (Matsuki et al., 2010) and the effect of elbow position on DRUJ kinematics (Fu et al., 2009) have been investigated.

In vitro testing of the DRUJ kinematics allows to set different conditions on a same cadaver such as sequential dissection of anatomy and sectioning of soft tissues, however, non-physiological loading and passive motion should be taken into consideration. Although in vivo techniques using CT imaging or an image-matching method have been validated to have adequate accuracy and precision (Fischer, et al., 2001; Li, et al., 2004), their application has still been limited. Thus the effects of elbow position and sex on the DRUJ kinematics have not been fully documented based on in vivo measurements where 3D bony movements can be determined directly.

The aim of the present study was to explore the in vivo 3D kinematics of the DRUJ during forearm pronation-supination of normal subjects in order to help understand the physiological biomechanics of the forearm. Using a 2D to 3D image matching method, we tested hypotheses that elbow flexion and sex of the subject affect the 3D kinematics of the DRUJ.

\section{Materials and methods}

After institutional review board approval and informed consent were obtained for each subject, 12 subjects (6 men, age 22-45, mean age 31 years, and 6 women, age 22-44, mean age 30.8 years) were enrolled in this study. Twelve right-dominant forearms without a history of disease or trauma were imaged by a CT scanner (BrightSpeed ${ }^{\mathrm{TM}}$ Elite SD; GE Healthcare, CT, USA) with a slice thickness of $0.63 \mathrm{~mm}$ and a reconstruction interval of $0.31 \mathrm{~mm}$. The tube current for the CT scan was limited to $20 \mathrm{~mA}$ to lower the radiation dose and the Adaptive Statistical Iterative Reconstruction technology (Hara, et al., 2009) was utilized for noise reduction. The CT scan data of each subject was imported into modeling software (ZedView ${ }^{\circledR}$ 4; LEXI, Tokyo, Japan) to create 3D surface models of the ulna and radius with the accuracy and precision of $0.40 \pm 0.33 \mathrm{~mm}$, determined by comparing the dimensions of the surface model with actual values (Sasagawa, et al., 2008). Each subject was then imaged using a biplanar x-ray system. This system consists of 2 $\mathrm{x}$-ray tubes (RADspeed Pro ${ }^{\mathrm{TM}}$ and MobileArt Evolution; Shimadzu, Kyoto, Japan) to allow frontal and lateral images to be taken with a one-second interval between the two exposures. Two $352 \mathrm{~mm} \times 428 \mathrm{~mm}$ imaging plates were used to capture the entire forearm (elbow to wrist) and digitized in 8-bit gray intensity with $0.1 \mathrm{~mm}$ resolution (FCR; Fuji, Tokyo, Japan). The subjects were seated and placed their right upper limbs on a radiolucent spacer with $90^{\circ}$ of flexion at the shoulder and full extension at the elbow (Fig. 1(a)). The subject rotated the forearm from $90^{\circ}$ of supination through $45^{\circ}$ of supination, neutral, $45^{\circ}$ of pronation, and $90^{\circ}$ of pronation. At each position, the forearm was held for a few seconds to be imaged. Next, the subject flexed $70^{\circ}$ at the elbow and $20^{\circ}$ at the shoulder to maintain the forearm in a horizontal position (Fig. 1(b)). Again, biplanar images were serially acquired from $90^{\circ}$ of supination to $90^{\circ}$ of pronation using the same procedure mentioned above. To calibrate the biplanar imaging system, an acrylic frame with 37 radiopaque markers was placed over the forearm.

The local coordinate systems were defined for the ulnar and radial models. The origin of the local coordinate system was placed at the center of the distal articular surface of each bone model. The long axis of the bone model determined by the principal component analysis (Kai, et al., 2014) was defined as the z-axis to represent proximodistal movement (distal direction was positive). The x-axis was set parallel to the line connecting the origin of the coordinate system and the radial or ulnar styloid process to represent radioulnar movement (ulnar direction was positive). The $\mathrm{y}$-axis was set to direct the palmar direction to represent dorsopalmar movement (palmar direction was positive). The 3 axes were set to be mutually orthogonal to describe the kinematics of the DRUJ as 6 degree-of-freedom parameters (Fig. 2). The lengths of the ulna and radius were measured as the distance between the most proximal and distal points along the longitudinal axis of the bone model.

Three-dimensional positions of the ulna and radius were determined using the 2D-to-3D image-matching technique. The accuracy of $0.5 \mathrm{~mm}$ for translation and $0.6^{\circ}$ for rotation was reported based on experiments using 
femoral and tibial 3D models (Kobayashi et al., 2009). The calibrated biplanar imaging system was replicated using custom-made software. The 3D ulna and radius models were imported into the software to allow them to be matched automatically with the biplanar radiographic images (Fig. 3). The relative position of the radius with respect to the ulna was determined in terms of the translations in the radioulnar, dorsopalmar, and proximodistal directions, as well as in terms of the rotations about the radioulnar, dorsopalmar, and proximodistal axes. Deviation from the neutral position was evaluated for each of the 6 parameters.

One-way analysis of variance (ANOVA) using the Bonferroni post-hoc test was carried out to compare the kinematics data. The effects of elbow position and sex were analyzed by paired $t$-tests and unpaired $t$-tests, respectively (SPSS 20; IBM, NY, USA). The level of significance was set at $P<0.05$.

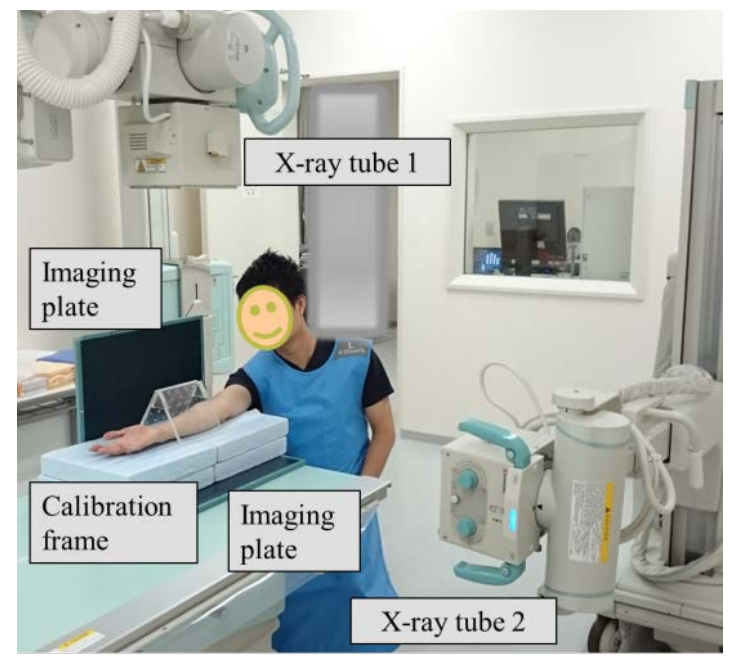

(a)

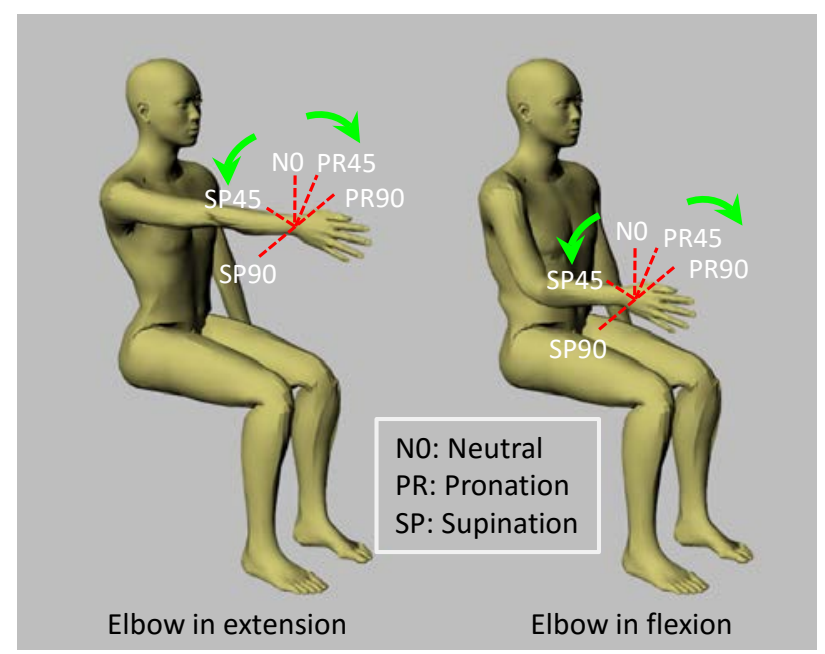

(b)

Fig. 1 Subject during biplanar X-ray imaging with the elbow in extension (a), and forearm positions to be measured (b).

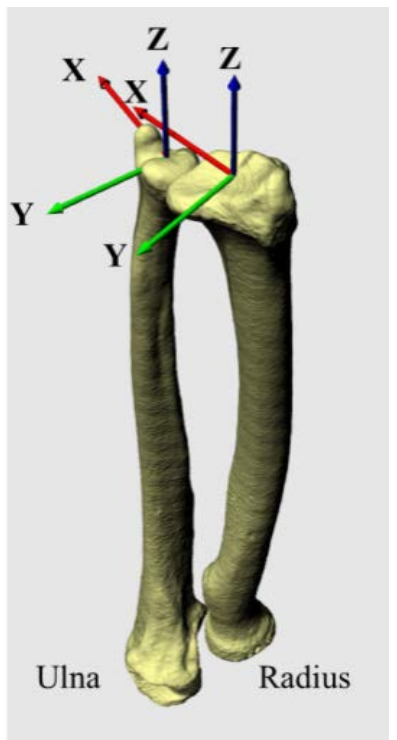

Fig. 2 Cartesian coordinate systems for the radius and ulna 


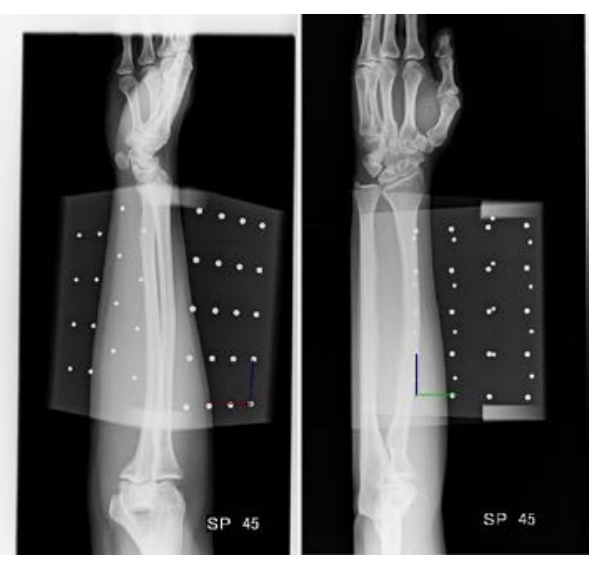

(a) Bi-plane X-ray images

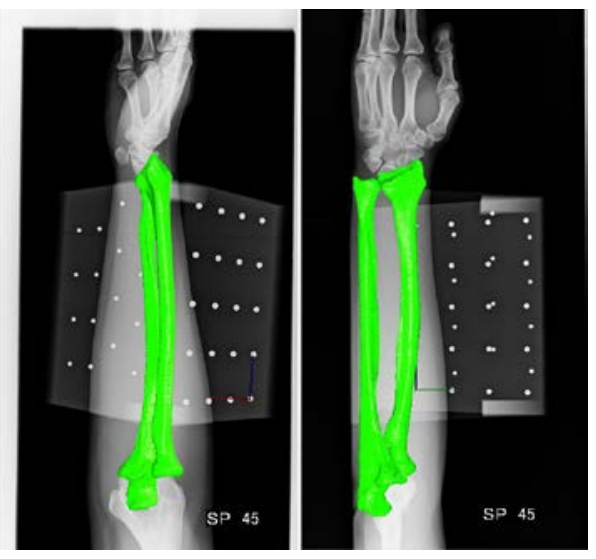

(b) Bone models overlaid

Fig. 3 Result of automated image matching of the radius and ulna at $45^{\circ}$ of supination

\section{Results}

\subsection{Bone length}

The mean lengths of the ulna and radius for men were both significantly greater than that for women $(272.6 \pm 13.8$ $\mathrm{mm}$ vs $232.8 \pm 15.6 \mathrm{~mm}$ for the ulna $[P<0.001]$ and $256.8 \pm 15.9 \mathrm{~mm}$ vs $217.5 \pm 17.3 \mathrm{~mm}$ for the radius $[P<0.001])$. All male subjects had longer ulnae and radiuses than any of the female subjects.

\subsection{Translation}

The relative translations of the radius with respect to the ulna in elbow extension and flexion are shown for both male and female subjects in Fig. 4. Note that marks $a$ and $b$ indicate significant differences between elbow extension and flexion, between male and female subjects to be described in section 3.4. The significance probabilities among the relative translations at the five forearm positions were given in Table 1.

Generally, the radius translated towards the ulnar side throughout forearm rotation, and moved towards the palmar side as pronation increased, whereas it moved dorsally as supination increased, and was positioned proximally during pronation and distally during supination for both male and female subjects, regardless of elbow position. For men, with the elbow in extension, translation of the radius to the ulnar side at $90^{\circ}$ of supination was significantly greater than those at $45^{\circ}$ of supination, neutral, and $45^{\circ}$ of pronation. The palmar translations were significantly different among forearm rotations except between $90^{\circ}$ and $45^{\circ}$ of pronation. On the other hand, there were no significant differences in proximal-distal translations among forearm rotations. With the elbow in flexion, the result was similar with that for the elbow in extension.

For women, with the elbow in extension, the translations of the radius to the ulnar side at $90^{\circ}$ of pronation and at supination were significantly greater than that at neutral. Dorsal and palmar translations were both significantly greater than that at neutral during pronation and supination. The radius translated distally at supination compared with that at $90^{\circ}$ of pronation. With the elbow in flexion, translation to the ulnar side was significantly greater than neutral throughout forearm rotation, except at $45^{\circ}$ of supination. Dorsal and palmar translations were both significantly greater than that at neutral during pronation and supination. The radius translated distally at $90^{\circ}$ of supination compared with those at neutral and $45^{\circ}$ of pronation.

\subsection{Rotation}

The relative rotations of the radius with respect to the ulna about the radioulnar and dorsopalmar axes were within $10^{\circ}$ for both male and female subjects, regardless of elbow position (Fig. 5). For men, with the elbow in extension, significant differences were found in the rotations about the dorsopalmar axis between supination and pronation. With the elbow in flexion, there were no significant differences in rotations about the radioulnar and dorsopalmar axes. Compared with neutral, rotations about the proximodistal axis were significantly different during pronation and supination, regardless of elbow position (Table 2).

For women, with the elbow in extension, significant differences were found in the rotations about the dorsopalmar axis between pronation and supination. With the elbow in flexion, compared with $90^{\circ}$ of supination, the rotations about the dorsopalmar axis were significantly different at neutral and pronation. Compared with neutral, the rotation about the proximodistal axis was significantly affected by pronation and supination, regardless of the elbow position (Table 2). 

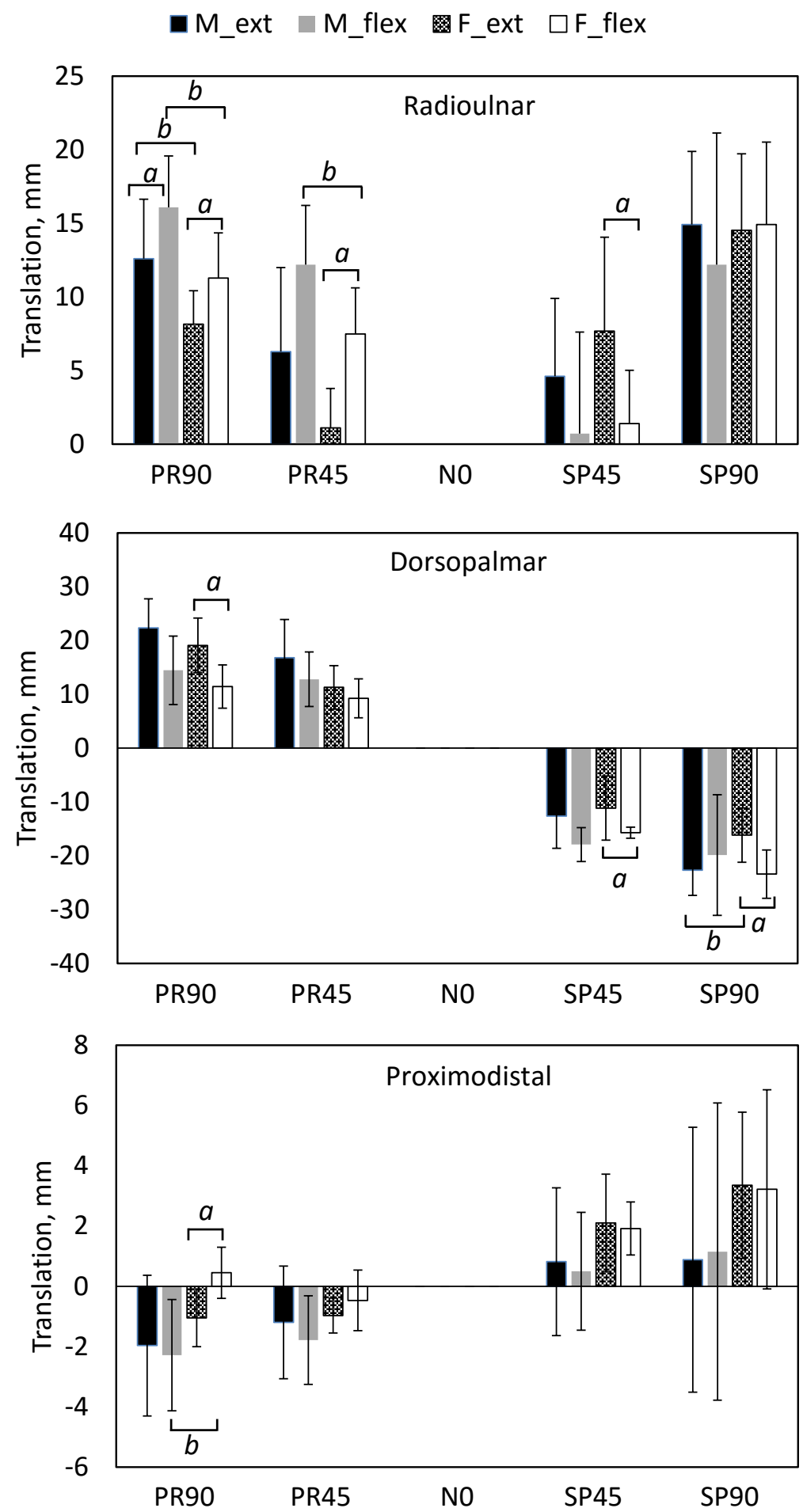

Fig. 4 Relative translation of the radius with respect to the ulna.

Abbreviations: M, male subjects; F, female subjects; ext, elbow in extension; flex, elbow in flexion; PR, pronation; N, neutral; SP, supination; $a$, significant difference between elbow extension and flexion; $b$, significant difference between male and female subjects. 

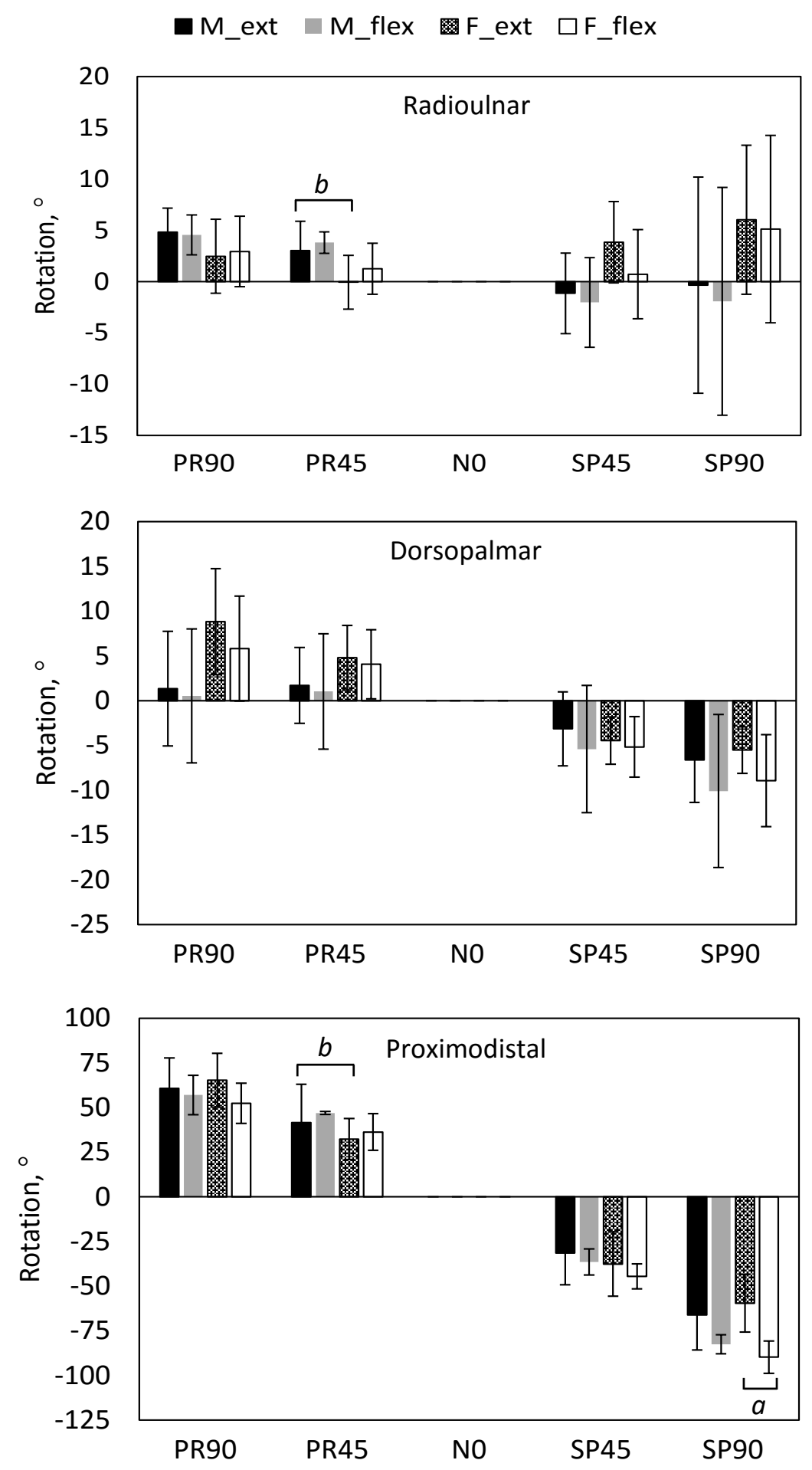

Fig. 5 Relative rotation of the radius with respect to the ulna.

M, male subjects; F, female subjects; ext, elbow in extension; flex, elbow in flexion; PR, pronation; N, neutral; SP, supination; $a$, significant difference between elbow extension and flexion; $b$, significant difference between male and female subjects. 


\subsection{Effects of 2 variables: elbow position and sex of the subjects}

Again, marks $a, b$, and $c$ indicate significant differences between elbow extension and flexion, between male and female subjects, and between male and female subjects after normalizing for length of the radius. For men, the effect of elbow position was found only with translation along the radioulnar direction with $90^{\circ}$ of pronation $(P<0.01)$. In contrast, for women, significant differences between elbow extension and flexion were observed with radioulnar translations at $90^{\circ}$ of pronation $(P<0.02), 45^{\circ}$ of pronation $(P<0.001), 45^{\circ}$ of supination $(P<0.02)$; dorsopalmar translations at $90^{\circ}$ of pronation $(P<0.05), 45^{\circ}$ of supination $(P<0.02)$ and $90^{\circ}$ of supination $(P=0.05)$; proximodistal translations at $90^{\circ}$ of pronation $(P<0.01)$, and rotation about the proximodistal axis at $90^{\circ}$ of pronation $(P<0.05)$.

Radioulnar translation at $90^{\circ}$ of pronation was significantly greater in men than in women with the elbow in extension $(P<0.05)$ and flexion $(P<0.03)$, and radioulnar translation at $45^{\circ}$ of pronation was significantly higher in men with elbow flexion $(P<0.05)$. Dorsopalmar translation at $90^{\circ}$ of supination was significantly greater in men $(P<$ 0.05). Proximal translation of the radius was greater in men than in women at $90^{\circ}$ of pronation $(P<0.01)$. After the translation of the radius was normalized by its length (Fig. 6), the significant difference between male and female subjects was found only with proximodistal translation at $90^{\circ}$ of pronation with the elbow in flexion $(P<0.01)$.

Rotations about the radioulnar axis and about the proximodistal axis were both greater in men at $45^{\circ}$ of pronation with the elbow in flexion $(P<0.05$ and $P<0.03$, respectively). 

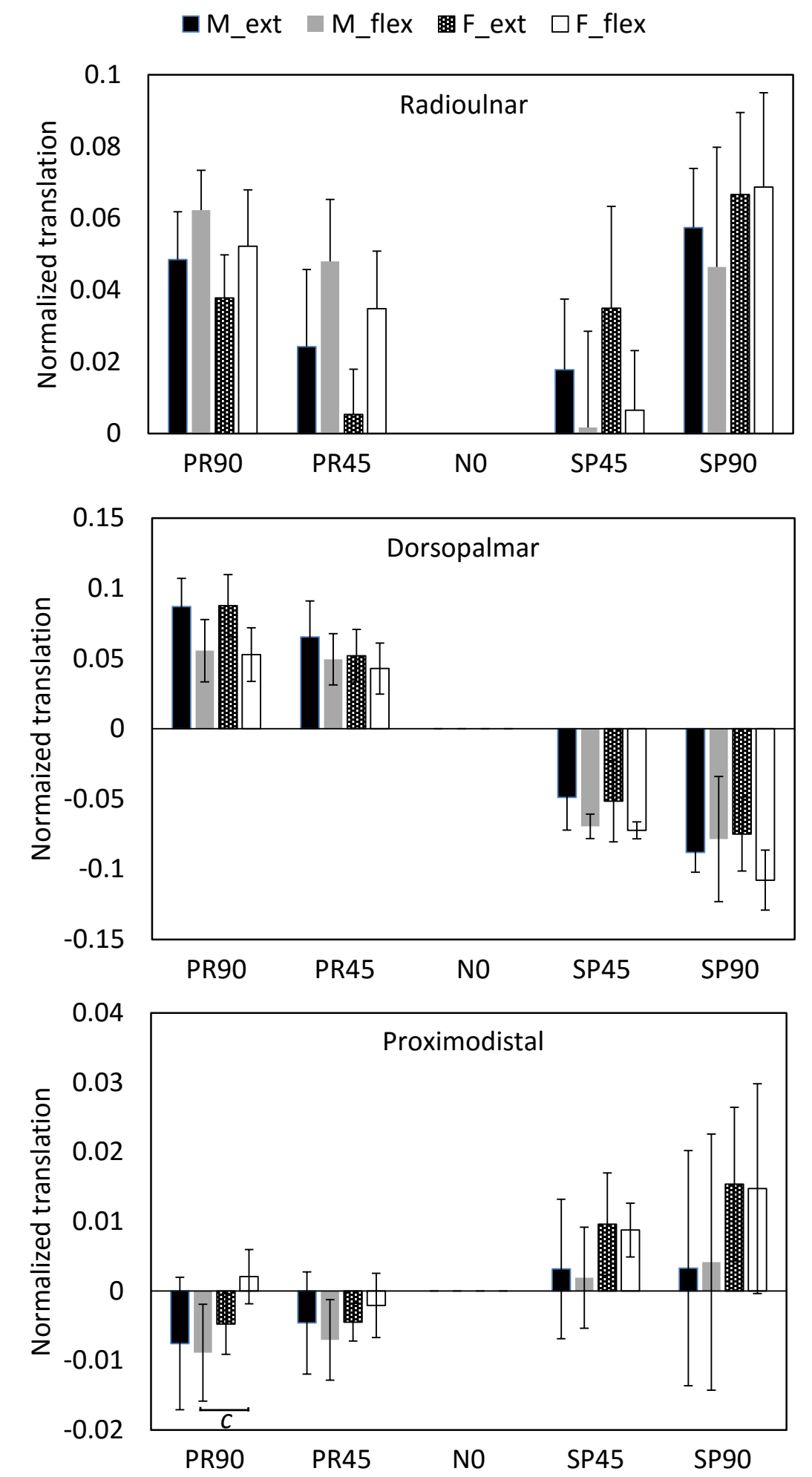

Fig. 6 Relative translation of the radius with respect to the ulna after normalizing for length of the radius. Abbreviations: M, male subjects; F, female subjects; ext, elbow in extension; flex, elbow in flexion; PR, pronation; N, neutral; SP, supination; $c$, significant difference between male and female subjects. 
Table 1 Significance probabilities among relative translations at the five forearm positions.

(a) Male subjects with the elbow in extension

\begin{tabular}{|c|c|c|c|c|c|}
\hline \multicolumn{6}{|c|}{ Radioulnar } \\
\hline \multicolumn{2}{|r|}{ PR90 } & \multirow{2}{*}{\multicolumn{2}{|c|}{$\begin{array}{cl}\text { PR45 } & \text { N0 } \\
\text { n.s. } & .001\end{array}$}} & \multirow{2}{*}{$\begin{array}{c}\text { SP45 } \\
\text { n.s. }\end{array}$} & \multirow{2}{*}{$\frac{\text { SP90 }}{\text { n.s. }}$} \\
\hline PR90 & & & & & \\
\hline PR45 & - & \multirow{2}{*}{-} & n.s. & \multirow{3}{*}{$\begin{array}{l}\text { n.s. } \\
\text { n.s. }\end{array}$} & \multirow{2}{*}{$\begin{array}{l}.028 \\
.000\end{array}$} \\
\hline N0 & - & & & & \\
\hline SP45 & - & \multirow{2}{*}{$\begin{array}{l}- \\
-\end{array}$} & - & & \multirow{2}{*}{.005} \\
\hline SP90 & - & & - & & \\
\hline \multicolumn{6}{|c|}{ Radioulnar } \\
\hline & \multicolumn{2}{|c|}{ PR90 PR45 } & N0 & SP45 & SP90 \\
\hline PR90 & $x$ & \multicolumn{2}{|c|}{ n.s. .000} & & n.s. \\
\hline PR45 & - & \multirow[b]{2}{*}{-} & .009 & .015 & \multirow{2}{*}{ n.s. } \\
\hline N0 & - & & & \multirow{2}{*}{ 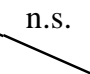 } & \\
\hline SP45 & - & - & - & & $\begin{array}{l}.009 \\
.015\end{array}$ \\
\hline SP90 & - & & - & \\
\hline \multicolumn{6}{|c|}{ Radioulnar } \\
\hline & PR90 & PR45 & N0 & SP45 & SP90 \\
\hline PR90 & & \multirow{2}{*}{\multicolumn{2}{|c|}{ n.s. .016}} & n.s. & n.s. \\
\hline PR45 & - & & & n.s. & .000 \\
\hline N0 & - & - & & .027 & .000 \\
\hline SP45 & - & - & - & & n.s. \\
\hline SP90 & - & - & - & - & \\
\hline & & Radi & ulnar & & \\
\hline & PR90 & PR45 & N0 & SP45 & SP90 \\
\hline PR90 & & n.s. & .000 & .001 & n.s. \\
\hline PR45 & - & & .013 & n.s. & .014 \\
\hline N0 & - & - & & n.s. & .000 \\
\hline SP45 & - & - & - & & .000 \\
\hline SP90 & - & - & - & - & \\
\hline
\end{tabular}

\begin{tabular}{|c|c|c|c|c|c|c|c|c|c|}
\hline \multicolumn{5}{|c|}{ Dorsopalmar } & \multicolumn{5}{|c|}{ Proximodistal } \\
\hline & PR90 PR45 & N0 & SP45 & SP90 & & PR90 PR45 & N0 & SP45 & SP90 \\
\hline$\overline{P R 90}$ & n.s. & .000 & .000 & .000 & PR90 & n.s. & n.s. & n.s. & n.s. \\
\hline PR45 & - & .000 & .000 & .000 & PR45 & - & n.s. & n.s. & n.s. \\
\hline N0 & - & & .003 & .000 & N0 & - & & n.s. & n.s. \\
\hline SP45 & - & - & & .027 & SP45 & - & - & & n.s. \\
\hline SP90 & - & - & - & & SP90 & - & - & - & \\
\hline \multicolumn{10}{|c|}{ (b) Male subjects with the elbow in flexion } \\
\hline \multicolumn{5}{|c|}{ Dorsopalmar } & \multicolumn{5}{|c|}{ Proximodistal } \\
\hline & PR90 PR45 & N0 & SP45 & SP90 & & PR90 PR45 & N0 & SP45 & SP90 \\
\hline PR90 & n.s. & .006 & .000 & .000 & PR90 & n.s. & n.s. & n.s. & n.s. \\
\hline PR45 & - & .018 & .000 & .000 & PR45 & - & n.s. & n.s. & n.s. \\
\hline No & - & & .000 & .000 & N0 & - & & n.s. & n.s. \\
\hline SP45 & - & - & & n.s. & SP45 & - & - & & n.s. \\
\hline SP90 & - & - & - & & SP90 & - & - & - & \\
\hline
\end{tabular}

(c) Female subjects with the elbow in extension

\begin{tabular}{|c|c|c|c|c|c|c|c|c|c|c|c|}
\hline \multicolumn{6}{|c|}{ Dorsopalmar } & \multicolumn{6}{|c|}{ Proximodistal } \\
\hline & PR90 & PR45 & N0 & SP45 & SP90 & & PR90 & PR45 & N0 & SP45 & SP90 \\
\hline PR90 & & n.s. & .000 & .000 & .000 & PR90 & & n.s. & n.s. & .006 & .000 \\
\hline PR45 & - & & .002 & .000 & .000 & PR45 & - & & n.s. & .008 & .000 \\
\hline N0 & - & - & & .003 & .000 & N0 & - & - & & n.s. & .003 \\
\hline SP45 & - & - & - & & n.s. & SP45 & - & - & - & & n.s. \\
\hline SP90 & - & - & - & - & & SP90 & - & - & - & - & \\
\hline
\end{tabular}

(d) Female subjects with the elbow in flexion

\begin{tabular}{|c|c|c|c|c|c|}
\hline \multicolumn{6}{|c|}{ Dorsopalmar } \\
\hline & PR90 & PR45 & N0 & SP45 & SP90 \\
\hline PR90 & & n.s. & .000 & .000 & .000 \\
\hline PR45 & - & & .000 & .000 & .000 \\
\hline N0 & - & - & & .000 & .000 \\
\hline SP45 & - & - & & & .003 \\
\hline SP90 & - & - & - & - & \\
\hline
\end{tabular}

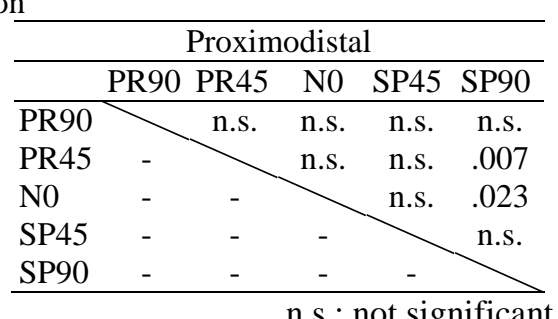

Table 2 Significance probabilities among relative rotations at the five forearm positions.

(a) Male subjects with the elbow in extension

\begin{tabular}{|c|c|c|c|c|c|}
\hline \multicolumn{6}{|c|}{ Radioulnar } \\
\hline & PR90 & PR45 & N0 & SP45 & SP90 \\
\hline PR90 & & n.s. & n.s. & n.s. & n.s. \\
\hline PR45 & - & & n.s. & n.s. & n.s. \\
\hline N0 & - & - & & n.s. & n.s. \\
\hline SP45 & - & - & - & & n.s. \\
\hline SP90 & - & - & - & - & \\
\hline
\end{tabular}

\begin{tabular}{|c|c|c|c|c|c|}
\hline \multicolumn{6}{|c|}{ Radioulnar } \\
\hline & PR90 & PR45 & N0 & SP45 & SP90 \\
\hline PR90 & 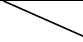 & n.s. & n.s. & n.s. & n.s. \\
\hline PR45 & - & & n.s. & n.s. & n.s. \\
\hline N0 & - & - & & n.s. & n.s. \\
\hline SP45 & - & - & - & & n.s. \\
\hline SP90 & - & - & - & - & \\
\hline
\end{tabular}

\begin{tabular}{|c|c|c|c|c|c|}
\hline \multicolumn{6}{|c|}{ Dorsopalmar } \\
\hline & PR90 & PR45 & N0 & SP45 & SP90 \\
\hline PR90 & & n.s. & n.s. & n.s. & .047 \\
\hline PR45 & - & & n.s. & n.s. & .032 \\
\hline N0 & - & - & & n.s. & n.s. \\
\hline SP45 & - & - & & & n.s. \\
\hline SP90 & - & - & - & - & \\
\hline
\end{tabular}

\begin{tabular}{lrrrrr}
\hline \multicolumn{5}{c}{ Proximodistal } \\
\hline & PR90 & PR45 & N0 & SP45 & SP90 \\
PR90 & & n.s. & .000 & .000 & .000 \\
PR45 & - & & .003 & .000 & .000 \\
N0 & - & - & & .036 & .000 \\
SP45 & - & - & - & & .017 \\
SP90 & - & - & - & - & \\
\hline
\end{tabular}

(b) Male subjects with the elbow in flexion

\section{Dorsopalmar}

\begin{tabular}{llcccc}
\hline \multicolumn{7}{r}{ PR90 } & PR45 & N0 & SP45 & SP90 \\
\hline PR90 & & n.s. & n.s. & n.s. & n.s. \\
PR45 & - & & n.s. & n.s. & n.s. \\
N0 & - & - & & n.s. & n.s. \\
SP45 & - & - & - & & n.s. \\
SP90 & - & - & - & - &
\end{tabular}

\begin{tabular}{lrrrrr}
\hline \multicolumn{5}{c}{ Proximodistal } \\
\hline & PR90 & PR45 & N0 & SP45 & SP90 \\
PR90 & & n.s. & .000 & .000 & .000 \\
PR45 & - & & .000 & .000 & .000 \\
N0 & - & - & & .000 & .000 \\
SP45 & - & - & - & & .000 \\
SP90 & - & - & - & - & \\
\hline
\end{tabular}

(c) Female subjects with the elbow in extension

\begin{tabular}{|c|c|c|c|c|c|}
\hline \multicolumn{6}{|c|}{ Radioulnar } \\
\hline & PR90 & PR45 & N0 & SP45 & SP90 \\
\hline PR90 & 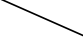 & n.s. & n.s. & n.s. & n.s. \\
\hline PR45 & - & & n.s. & n.s. & n.s. \\
\hline N0 & - & - & & n.s. & n.s. \\
\hline SP45 & - & - & - & & n.s. \\
\hline SP90 & - & - & - & - & \\
\hline
\end{tabular}

\begin{tabular}{|c|c|c|c|c|c|}
\hline \multicolumn{6}{|c|}{ Dorsopalmar } \\
\hline & PR90 & PR45 & N0 & SP45 & SP90 \\
\hline PR90 & 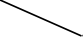 & n.s. & .002 & .000 & .000 \\
\hline PR45 & - & & n.s. & .001 & .000 \\
\hline N0 & - & - & & n.s. & n.s. \\
\hline SP45 & - & - & - & & n.s. \\
\hline SP90 & - & - & - & - & \\
\hline
\end{tabular}

\begin{tabular}{lrrrrr}
\hline \multicolumn{5}{c}{ Proximodistal } \\
\hline & PR90 & PR45 & N0 & SP45 & SP90 \\
PR90 & & .003 & .000 & .000 & .000 \\
PR45 & - & & .004 & .000 & .000 \\
N0 & - & - & & .001 & .000 \\
SP45 & - & - & - & & n.s. \\
SP90 & - & - & - & - & \\
\hline
\end{tabular}


(d) Female subjects with the elbow in flexion

\begin{tabular}{|c|c|c|c|c|c|}
\hline \multicolumn{6}{|c|}{ Radioulnar } \\
\hline & PR90 & PR45 & N0 & SP45 & SP90 \\
\hline PR90 & & n.s. & n.s. & n.s. & n.s. \\
\hline PR45 & - & & n.s. & n.s. & n.s. \\
\hline N0 & - & - & & n.s. & n.s. \\
\hline SP45 & - & - & - & & n.s. \\
\hline SP90 & - & - & - & - & \\
\hline
\end{tabular}

\begin{tabular}{|c|c|c|c|c|c|}
\hline \multicolumn{6}{|c|}{ Dorsopalmar } \\
\hline & PR90 & PR45 & N0 & SP45 & SP90 \\
\hline PR90 & & n.s. & n.s. & .001 & .000 \\
\hline PR45 & - & & n.s. & .008 & .000 \\
\hline N0 & - & - & & n.s. & .011 \\
\hline SP45 & - & - & - & & n.s. \\
\hline SP90 & - & - & - & - & \\
\hline
\end{tabular}

\begin{tabular}{|c|c|c|c|c|}
\hline \multicolumn{5}{|c|}{ Proximodistal } \\
\hline & PR90 PR45 & N0 & SP45 & SP90 \\
\hline PR90 & $\times .003$ & .000 & .000 & .000 \\
\hline PR45 & - & .000 & .000 & .000 \\
\hline N0 & - & & .000 & .000 \\
\hline SP45 & - & - & & .000 \\
\hline SP90 & - & - & - & \\
\hline
\end{tabular}

\section{Discussion}

In this study, we investigated the in vivo 3D kinematics of the DRUJ during forearm pronation and supination of normal subjects. The relative translation and rotation of the radius with respect to the ulna were examined with the elbow in extension and flexion. The kinematics data were compared between the male and female subjects.

Our first hypothesis that elbow flexion affects the 3D kinematics of the DRUJ was supported as the translations along the radioulnar, dorsopalmar, and proximodistal directions and the rotation about the proximodistal axis changed with elbow flexion. Our second hypothesis that the kinematics of DRUJ differs with sex was also supported while the sex differences were suppressed mostly after accounting for the length of the radius.

Previous in vivo studies using 2D-to-3D image-matching methods using single or biplanar fluoroscopy have revealed that the ulnar head translated, relative to the radius, dorsally as the forearm rotated from maximum supination to maximum pronation (Matsuki, et al., 2010), and the position of the radius relative to the ulna was proximal during pronation and distal during supination, and this was affected by elbow flexion at mid-pronation (Fu, et al., 2009). Using CT scans, Tay et al. (2010) and Chen and Tang (2013) also reported that the radius translated proximally during pronation and distally during supination. In general, our results were consistent with in vivo data reported in the literature while the effect of elbow position on proximal-distal translation was found only for female subjects. Because the elbow position affects the restriction of soft tissues to bony movements, the DRUJ kinematics were changed with the elbow in extension and flexion. However, the extent of changes with the elbow position on the DRUJ kinematics varied with the degree of pronation and supination, indicating that the stabilizing effect of the soft tissues became more complicated with the change in elbow position. This should be considered in the development of a relevant musculoskeletal model of the forearm.

Sex differences in forearm kinematics have been examined based on gross movement measurements (Rickert, et al., 2008; Soucie, et al., 2010), indicating that larger ROMs of pronation and supination were found for female subjects than for male subjects. As we investigated the kinematics at the prescribed pronation and supination positions (not at maximum pronation or maximum supination), the ROM was the same for both male and female subjects, while the relative movements of the radius on the ulna were generally greater in women. One possible explanation for this discrepancy is that the restriction of the soft tissues to bony movements was less in women. This could result in a larger ROM from maximum pronation to maximum supination in women as reported previously.

Regarding the sex difference, the discrepancy in bone length between the male and female subjects should be considered. Rainbow et al. (2008) suggested that sex differences in capitate kinematics were eliminated after accounting for carpal size. Our data partially supported this result because, after normalizing the translation for length of the radius, the sex difference was eliminated, except during proximodistal translation at $90^{\circ}$ of pronation with the elbow in flexion. However, because other anatomical factors such as ulnar foveal shape have an impact on the kinematics of DRUJ motion (Kataoka, et al., 2012), the effects of the geometry as well as the shape of the bones on forearm kinematics should be further investigated.

Ulnar variance has been considered to be one of the causes of dysfunction at the DRUJ. Saito et al (2014) pointed out that, relative to the radius, the ulnar head positioned dorsally and distally during pronation (plus variant), while positioned palmarly and proximally during supination (minus variant). This is supported by previous in vitro (Quigley, et al., 2013) and in vivo (Fu, et al., 2009, Tay, et al., 2010, Chen and Tang, 2013) reports. Our study also showed that, in general, the radius translated proximally during pronation and distally during supination relative to the ulna. Moreover, the effects of elbow position and sex (or the length of the radius) were found in the proximodistal translation at $90^{\circ}$ of pronation. Clinically, ulnar variance is evaluated on a $2 \mathrm{D}$ anteroposterior radiograph with the wrist in neutral forearm rotation, the elbow in $90^{\circ}$ of flexion, and the shoulder in $90^{\circ}$ of abduction. In addition to the previous findings, the current results suggest that ulnar variance can vary with elbow flexion as well as with regard to sex apart from the neutral forearm position.

As for the radiation dose issue, this study has an advantage over previous studies using CT scans. Tay et al. (2010) performed CT scans 5 times for each subject with a tube voltage of $120 \mathrm{kV}$ and a tube current-time product of 110 mAs. In this study, CT scan with $120 \mathrm{kV}$ and $20 \mathrm{mAs}$ was carried out only once for each subject. Thus, the radiation dose was considerably lower, even after 10 radiographs (50 kV and $0.28 \mathrm{mAs}$ ) by biplanar imaging were added. 
Another advantage of this study was that our biplanar imaging system had a larger field of view than traditional fluoroscopes. The receiver of common fluoroscopes is an approximately $310 \mathrm{~mm}$ diameter circle, with which it is impossible to capture the entire forearm (elbow to wrist). The image size of the current study, $352 \mathrm{~mm} \times 428 \mathrm{~mm}$, allows for imaging the whole forearm structure and analyzing the kinematics of the PRUJ in addition to the DRUJ.

There are limitations in the current study. The forearm was imaged in static rotation. Further study should examine pronation and supination during dynamic rotation of the forearm. Additionally, the relatively small sample size may have biased the kinematics results.

\section{Conclusions}

In this study, we investigated the in vivo 3D kinematics of the DRUJ during forearm pronation and supination of normal subjects. The radius translated towards the ulnar side throughout forearm rotation and moved towards the palmar side as pronation increased, whereas it moved dorsally as supination increased and was positioned proximally during pronation and distally during supination. The rotation of the radius was dominant about the proximodistal axis. These kinematic variables were affected by both elbow position and sex of the subject. These results could contribute to understanding the physiological biomechanics of the DRUJ.

\section{Acknowledgments}

The authors wish to thank Dr. Yutaka Maki, MD, PhD and Dr. Naoto Tsubokawa, MD, PhD of Niigata Hand Surgery Foundation, Niigata Hand Care Center for providing the facilities for experimentation.

\section{References}

Chen, Y.R. and Tang, J.B., In vivo gliding and contact characteristics of the sigmoid notch and the ulna in forearm rotation, Journal of Hand Surgery, Vol.38A (2013), pp.1513-1519.

Fischer, K.J., Manson, T.T., Pfaeffle, H.J., Tomaino, M.M., Woo, S.L.Y., A method for measuring joint kinematics designed for accurate registration of kinematic data to models constructed from CT data, Journal of Biomechanics, Vol.34(2001), pp.377-383.

Fu, E., Li, G., Souer, J.S., Lozano-Calderon, S., Herndon, J.H., Jupiter, J.B. and Chen, N.C., Elbow position affects distal radioulnar joint kinematics, Journal of Hand Surgery, Vol.34A (2009), pp.1261-1268.

Hara, A.K., Paden, R.G., Silva, A.C., Kujak, J.L., Lawder, H.J. and Pavlicek, W., Iterative reconstruction technique for reducing body radiation dose at CT: feasibility study, American Journal of Roentgenology Vol.193, No.3 (2009), pp.764-771.

Kai S., Sato T., Koga Y., Omori G., Kobayashi K., Sakamoto M., Tanabe Y., Automatic construction of an anatomical coordinate system for three-dimensional bone models of the lower extremities--pelvis, femur, and tibia, Journal of Biomechanics, Vol.47(2014), pp.1229-1233.

Kapandji, A., Biomechanics of pronation and supination of the forearm, Hand Clinics, Vol.17, No.1 (2001), pp.111-122.

Kataoka, T., Moritomo, H., Omokawa, S., Iida, A., Murase, T. and Sugamoto, K., Ulnar variance: Its relationship to ulnar foveal morphology and forearm kinematics, Journal of Hand Surgery, Vol.37A (2012), pp.729-735.

Kobayashi, K., Sakamoto, M., Tanabe, Y., Ariumi, A., Sato, T., Omori, G. and Koga, Y., Automated image registration for assessing three-dimensional alignment of entire lower extremity and implant position using bi-plane radiography. Journal of Biomechanics, Vol.42 (2009), pp.2818-2822.

Li, G., Wuerz T.H., DeFrate, L.E., Feasibility of using orthogonal fluoroscopic images to measure joint kinematics, Journal of Biomechanical Engineering, Vol.126 (2004), pp.314-318.

Matsuki, K.O., Matsuki, K., Mu, S., Sasho, T., Nakagawa, K., Ochiai, O., Takahashi, K. and Banks, S.A., In vivo 3D kinematics of normal forearms: Analysis of dynamic forearm rotation, Clinical Biomechanics, Vol.25 (2010), pp.979-983.

Nakamura, T., Yabe, Y., Horiuchi, Y. and Yamazaki, N., In vivo motion analysis of forearm rotation utilizing magnetic resonance imaging, Clinical Biomechanics, Vol.14, No.5 (1999), pp.315-320.

Quigley, R.J., Robicheaux, G,W, and Lee, T.Q., The proximal and distal position of the radius relative to the ulna through a full range of elbow flexion and forearm rotation, Journal Hand Surgery European Volume, Vol.39, No.5 (2013), pp.535-540.

Rainbow, M.J., Crisco, J.J., Moore, D.C. and Wolfe, S.W., Gender differences in capitate kinematics are eliminated after accounting for variation in carpal size, Transactions of the ASME, Journal of Biomechanical Engineering, Vol.130, No.4 (2008), 041003. doi:10.1115/1.2913332.

Ray, R.D., Johnson, R.T. and Jameson, R.M., Rotation of the forearm: An experimental study of pronation and supination, Journal of Bone and Joint Surgery, Vol.33A (1951), 993-996. 
Rickert, M., Bürger A., Günther, C.M. and Schulz, C.U., Forearm rotation in healthy adults of all ages and both sexes, Journal of Shoulder and Elbow Surgery, Vol.17, No.2 (2008), pp.271-275.

Saito, H., Yoshizu, T., Maki, Y., and Kanaya, F., Textbook of Hand Surgery, 2nd Edition (2014), pp.177-299, Nankodo. (in Japanese)

Sasagawa, K., Watanabe, S., Kobayashi, K., Sakamoto, M., Tanabe, Y., Sato, T. and Koga, Y., Accuracy examination of three-dimensional bone surface model using MRI and CT. Japanese Journal of Clinical Biomechanics, Vol.29 (2008), pp.397-402. (in Japanese)

Shaaban, H., Giakas, G., Bolton, M., Williams, R., Scheker, L.R. and Lees, V.C., The distal radioulnar joint as a load-bearing mechanism- a biomechanical study, Journal of Hand Surgery, Vol.29A (2004), pp.85-95.

Soucie, J.M., Wang, C., Forsyth, A., Funk, S., Denny, M., Roach, K.E. and Boone, D., Range of motion measurements: reference values and a database for comparison studies, Haemophilia, Vol.17 (2011), pp.500-507.

Stuart, P.R., Berger, R.A., Linscheid, R.L. and An, K-N., The dorsopalmar stability of the distal radioulnar joint, Journal of Hand Surgery, Vol.25A (2000), pp.689-699.

Tang, J.B. and Chen Y.R., In vivo changes in contact regions of the radiocarpal joint during wrist hyperextension, Journal of Hand Surgery, Vol.37A (2012), pp.2257-2262.

Tay, S.C., Berger, R.A., Kazunari, T., Tan, E.T., Amrami, K.K. and An, K-N., In vivo three-dimensional displacement of the distal radioulnar joint during resisted forearm rotation, Journal of Hand Surgery, Vol.32A (2007), pp.450-458.

Tay, S,C, van Riet, R., Kazunari, T., Amrami, K.K., An, K-N. and Berger, R.A., In-vivo kinematic analysis of forearm rotation using helical axis analysis, Clinical Biomechanics, Vol.25 (2010), pp.655-659. 